\title{
Numerical experiments on vortex ring formation
}

\author{
By KAMRAN MOHSENI, HONGYU RAN \\ AND TIM COLONIUS \\ Division of Engineering and Applied Sciences, 104-44, California Institute of Technology, \\ Pasadena, CA 91126, USA
}

(Received 17 December 1999 and in revised form 14 June 2000)

Numerical simulations are used to study the formation of vortex rings that are generated by applying a non-conservative force of long duration, simulating experimental vortex ring generation with large stroke ratio. For sufficiently long-duration forces, we investigate the extent to which properties of the leading vortex ring are invariant to the force distribution. The results confirm the existence of a universal 'formation number' defined by Gharib, Rambod \& Shariff (1998), beyond which the leading vortex ring is separated from a trailing jet. We find that the formation process is governed by two non-dimensional parameters that are formed with three integrals of the motion (energy, circulation, and impulse) and the translation velocity of the leading vortex ring. Limiting values of the normalized energy and circulation of the leading vortex ring are found to be around 0.3 and 2.0, respectively, in agreement with the predictions of Mohseni \& Gharib (1998). It is shown that under this normalization smaller variations in the circulation of the leading vortex ring are obtained than by scaling the circulation with parameters associated with the forcing. We show that by varying the spatial extent of the forcing or the forcing amplitude during the formation process, thicker rings with larger normalized circulation can be generated. Finally, the normalized energy and circulation of the leading vortex rings compare well with the same properties for vortices in the Norbury family with the same mean core radius.

\section{Introduction}

The generation, formation, evolution, and interactions of vortex rings have been the subject of numerous studies (e.g. Shariff \& Leonard 1992). In the laboratory, vortex rings can be generated by the motion of a piston pushing a column of fluid through an orifice or nozzle. The boundary layer at the edge of the orifice or nozzle will separate and roll up into a vortex ring. An important problem in vortex ring generation is to determine how much circulation and energy can be delivered to the vortex ring by the apparatus. Experiments (Gharib, Rambod \& Shariff 1998) have shown that for large piston stroke to diameter ratios, $L / D$, the generated flow field consists of a leading vortex ring followed by a trailing jet. The vorticity field of the leading vortex ring is disconnected from that of the trailing jet at a critical value of $L / D$ (dubbed the 'formation number'), at which time the vortex ring attains a maximum circulation. The formation number was in the range 3.6 to 4.5 for a variety of exit diameters, exit plane geometries, and non-impulsive piston velocities. An explanation for this effect was given based on the Kelvin-Benjamin variational 
principle, which states that steadily translating vortex rings have maximum energy with respect to isovortical perturbations that preserve impulse.

Mohseni \& Gharib (1998) considered a theoretical model to predict the formation number. They suggest that the properties of the leading vortex ring are the final outcome of a relaxational process, dependent only on the first few integrals of the motion, namely the energy, $E$, impulse, $I$, and circulation, $\Gamma$. While there is an infinite number of conserved integrals of motion in an inviscid flow, on a macroscopic level only the linear functionals of the vorticity density (energy, impulse and circulation) are conserved (Miller, Weichman \& Cross 1992; Mohseni 2000). These are, in fact, the integrals considered in the Kelvin-Benjamin variational principle.

They determine the pinch-off criterion as that time at which the integrals of motion delivered to the flow by the cylinder/piston arrangement (determined by resorting to a slug-flow model of the discharge process) are equivalent to those consistent with the one-parameter family of Norbury vortices. The parameter is the mean core radius, $\alpha_{N}$, which varies from zero for (infinitely) thin-cored rings to $\sqrt{2}$ for Hill's spherical vortex. In equation form, these statements are written

$$
\tilde{E}\left(\frac{t U_{t r}}{D}\right)=f\left(\alpha_{N}\right), \quad \tilde{\Gamma}\left(\frac{t U_{t r}}{D}\right)=g\left(\alpha_{N}\right),
$$

where

$$
\tilde{E}=\frac{E}{\Gamma^{3 / 2} I^{1 / 2}}, \quad \tilde{\Gamma}=\frac{\Gamma}{I^{1 / 3} U_{t r}^{2 / 3}} .
$$

$D, U_{t r}$ are the suitably defined toroidal diameter and translation velocity of the leading vortex ring. In most cases, the forward diameter will be very close to the cylinder diameter. The functional dependence on the right-hand sides of equation (1.1) is determined by the model for the leading vortex ring (Norbury family), while the functional dependence of the left-hand sides is determined by the generation apparatus. The values of $\alpha_{N}$ and $t U_{t r} / D$ which satisfy the two relations then give the thickness of the leading vortex ring, and the normalized pinch-off time, $t^{*}=t_{p o} U_{t r} / D$, respectively. For the Norbury/slug-flow model, Mohseni \& Gharib (1998) determined that $t^{*}$ was around 1.5 to 2.3 , which was equivalent to a stroke ratio, $L / D$, in the range of 3 to 4.6 . The range of values of $\tilde{\Gamma}$ and $\tilde{E}$ consistent with these pinch-off times were 1.77 to 2.07 and 0.27 to 0.4 , respectively. These values are consistent with experiments (Gharib et al. 1998) and computations (Rosenfeld, Rambod \& Gharib 1998; Zhao, Frankel, Mongean 2000) that have shown that vortex ring generators with different stroke length, orifice diameters, piston velocities, and orifice geometries all lead to similar formation numbers, so long as the velocity profile leaving the orifice is nearly uniform. Gharib et al. (1998) measured values of $\tilde{E}$ around 0.33 , and Rosenfeld et al. (1998) showed that in addition to the universality of the formation time, the circulation of the leading vortex ring, when scaled with the average piston velocity and cylinder diameter, varied by only $40 \%$ as the formation conditions were varied, though it turns out, as we show here, that normalizing the circulation with the impulse and translation velocity leads to a much better collapse of the data.

It would appear from the above that a limiting pinch-off time, $t^{*}$, would be a generic feature of vortex formation by other generation mechanisms. The functional dependence on the right-hand sides of equations (1.1) would presumably change if the apparatus delivers circulation, energy, and impulse to the flow at rates different from the cylinder/piston mechanism. In order to test this hypothesis and provide further data regarding the invariance of the normalized energy and circulation of 
the leading vortex ring, we have numerically computed vortex ring generation due to long-duration non-conservative body forces in the momentum equation. The details of the forcing are discussed in the next section. The computational method is briefly detailed in $\S 3$. In $\S 4.1$, we compare the flow field generated by long-duration forcing to previous results for the cylinder/piston mechanism of vortex ring generation. In $\S 4.2$ we vary parameters associated with the non-impulsive forcing, and examine the extent to which $t^{*}, \tilde{\Gamma}$, and $\tilde{E}$ are invariant to the parameters. The details of the computations allow us to construct a dynamic interpretation of the pinch-off process, which is discussed in $\S 4.3$. This interpretation leads to new ideas for generating thick vortex rings (with relatively higher normalized circulation); numerical experiments which successfully implement these ideas are discussed in $\S 4.4$. A comparison with Norbury vortices is given in $\S 4.5$. Concluding remarks are placed in $\S 5$.

\section{Vortex generation by non-conservative force}

There are several ways to model the vortex generation process. A given vorticity distribution and its corresponding velocity field may be used as the initial condition (Stanaway \& Cantwell 1988). A vortex sheet model was implemented by Nitsche \& Krasny (1994) to reproduce vortex generation experiments by Didden (1979) for small stroke ratios. James \& Madnia (1996) used numerical solutions of the low Mach number Navier-Stokes equations for small stroke ratios. They studied the formation, entrainment and mixing processes in vortex rings, as well as the effects of different vortex generators (namely, orifice versus nozzle) and velocity programs on the dynamics and kinematics of the resulting vortex rings. Another method is to prescribe an axial velocity profile $U_{x}(r)$ at an inlet, in an attempt to model the injection of fluid through the nozzle of the experimental apparatus (Verzicco et al. 1996). This approach was also used in recent computations (Rosenfeld et al. 1998; Zhao et al. 2000) that were discussed in the previous section.

An alternative method of numerically generating vortex rings is to apply a nonconservative force directly in the equations of motion. We view this as a 'generic' vortex ring generator, independent of any particular geometry, but note that in the laboratory, such a force could in principle be generated by imposing currents in the fluid with a magnetic field. This method has previously been used, for impulsive forces, in order to generate vortex rings with certain desired properties (McCormack \& Crane 1973; Swearingen, Crouch \& Handler 1995).

The body force is aligned with the axial coordinate of an axisymmetric cylindricalpolar coordinate system $(x, r)$ and has the form

$$
f_{x}(r, x, t)=C F(t) G(x) H(r)
$$

where $C$ is an amplitude constant with units of circulation, and $F, G$ are functions with units of inverse time and inverse length, respectively, and $H$ is a non-dimensional function.

For non-impulsive cases, we use a regularized step function for $F$ :

$$
F(t)=-\frac{\tanh \left(\alpha_{t}\left(t_{0}-t\right)\right)+\tanh \left(\alpha_{t}\left(t-t_{0}-T\right)\right)}{2 T}
$$

where $T$ is the duration of the force and $\alpha_{t}$ is a time scale which controls the smoothing. We have experimented with different values of $\alpha_{t}$, and the results presented below use $\alpha_{t}=2.0 R^{2} / C$, where $R$ is the radial extent of the forcing region. This is short enough that the vortex formation process was essentially independent of its value. 
For comparison, impulsive forcing can be obtained by setting $F(t)$ to a regularized Dirac delta function (a Gaussian):

$$
F_{\text {imp }}(t)=\frac{\exp -\left(\frac{t-t_{0}}{\alpha_{t}}\right)^{2}}{\alpha_{t} \sqrt{\pi}} .
$$

The functions $G$ and $H$ control the spatial variation of the flow generated by the force. For the radial distribution, we use

$$
H(r)=\frac{1}{2} \operatorname{erfc}\left(\sqrt{\alpha} \frac{r-R}{\alpha_{r}}\right)
$$

where $\alpha$ is a non-dimensional constant discussed below. This a smoothed 'top-hat' function, where $R$ is the radial extent of the forcing region, and $\alpha_{r}$ controls the smoothing of the profile; $\alpha_{r}$ is reminiscent of the boundary layer thickness at the exit of the nozzle in experiments. It controls the shear layer thickness of the resulting flow. For the axial distribution, we again use a regularized delta function:

$$
G(x)=\frac{\sqrt{\alpha}}{\alpha_{x} \sqrt{\pi}} \exp -\alpha\left(\frac{x}{\alpha_{x}}\right)^{2}
$$

where $\alpha_{x}$ controls the axial extent over which the force is smeared.

For impulsive forces, the azimuthal vorticity field generated by the force is given, to leading order (e.g. Saffman 1992), by

$$
\omega_{\theta}(x, r)=-\frac{\partial f_{x}(x, r)}{\partial r} .
$$

Thus for an impulsive force, the vorticity field is an approximately Gaussian distribution (for $\alpha_{r} \ll R$ ):

$$
\omega_{\theta}=\frac{C \alpha}{\pi \alpha_{x} \alpha_{r}} \exp -\alpha\left(\left(\frac{r-R}{\alpha_{r}}\right)^{2}+\left(\frac{x}{\alpha_{x}}\right)^{2}\right),
$$

which is the rationale for the specific functional dependence chosen for $H$ and $G$ given above, which we carry over to the non-impulsive case. Weigand \& Gharib (1997) showed that vortex rings generated by a piston/cylinder arrangement also possess an approximately Gaussian vorticity distribution in their core. Note that the constant factor $\alpha=1.25643 \ldots$ is used so that the maximum tangential velocity in the core is located at $r=R \pm \alpha_{r}$ when $\alpha_{r} \ll R$. Thus $\alpha_{r}$ is also a measure of the core radius. The ellipticity of the vorticity distribution in this case is controlled by $\alpha_{x}$. Clearly the circulation, for the impulsive force, is $\Gamma=C$.

Returning to the non-impulsive forcing given above, there are four independent non-dimensional parameters that may be formed: $\alpha_{r} / R, \alpha_{x} / R, T C / R^{2}$, and $R e=C / v$. By analogy with the cylinder/piston vortex generators, $T$ controls the stroke length. Larger $T$ corresponds to larger $L / D$. Therefore, vortex rings generated by small stroke ratio are modelled by impulsive forcing with small $T$, while vortex rings generated by large stroke ratio (e.g. pinched-off vortex rings) are modelled by larger $T$. For non-impulsive forces, $\Gamma \neq C$, and we therefore define an additional Reynolds number:

$$
R e_{l . v . r .}=\frac{\Gamma_{l . v . r .}}{v}, \quad \text { (non-impulsive force) }
$$

where $\Gamma_{l . . v . r}$ is the (measured) circulation of the leading vortex ring. 


\section{Numerical method}

For practical reasons, an existing code for the axisymmetric compressible NavierStokes equations has been used to study vortex ring formation. While the numerical method is inefficient for computing low Mach number flows, the CPU requirements for the present problem are minimal and all the computations were performed on a small workstation. In the cases presented here, the maximum Mach number in the flow was 0.2 , and it was verified that changes in Mach number below this value had no impact on the results presented.

A sixth-order compact finite difference scheme (Lele 1992) is used in both the axial and radial directions, and an explicit fourth-order Runge-Kutta method is used for time advancement. At the boundary we use a buffer zone (Freund 1997) to absorb the waves, and the polar coordinate centreline treatment proposed by Mohseni \& Colonius (2000) is used. From the location of the applied force, the computational domain extends to $-3 R$ and $21 R$ in the axial directions and about $4 R$ in the radial direction. The grid spacing is $\frac{1}{25} R$, and the time step is $\frac{1}{50} R / a$ where $a$ is the sound speed. Calculations with higher time and spatial resolutions established the grid independence of the solution.

For the results presented in the next section, we need to calculate the following integrals of the motion for the leading vortex ring:

$$
E=\pi \int \omega \psi \mathrm{d} x \mathrm{~d} r, \quad I=\pi \int \omega r^{2} \mathrm{~d} x \mathrm{~d} r, \quad \Gamma=\int \omega \mathrm{d} x \mathrm{~d} r .
$$

To do this, the velocity field is differentiated to obtain the vorticity. In order to distinguish between the leading vortex ring and the rest of the vorticity, we set the vorticity to zero outside a closed iso-contour of vorticity around the leading vortex core, at which point the vorticity is $2 \%$ of the maximum vorticity in the core. Then an integral equation (e.g. Saffman 1992) is used to obtain the stream function, $\psi$ from the vorticity distribution, and equations (3.1) are integrated numerically with a fourth-order-accurate quadrature scheme. The translational velocity, $U_{t r}$ is estimated by tracking the position of the maximum vorticity in the leading vortex core.

To estimate the uncertainty in determining $\tilde{E}$ and $\tilde{\Gamma}$ from the computational data in this way, we used the algorithm to compute the known integrals of motion for Hill's spherical vortex. With the present grid the errors in calculating $\Gamma, E, I$ are $0.5 \%, 4 \%$, $6 \%$ respectively. Another uncertainty in determining $\tilde{E}$ and $\tilde{\Gamma}$ comes from the cutoff level. As mentioned above, for all cases, we use $2 \%$ of the maximum vorticity in the core. The optimal cutoff level should be the minimum contour level that distinguishes between the leading vortex ring and the rest of the vorticity, which is varying with time and Reynolds number. For a few cases, we explicitly searched for the optimal value, and found that the $2 \%$ level led to differences in the integrals of about $1 \%$. The values quoted throughout the rest of the paper are for the $2 \%$ cutoff.

\section{Results}

\subsection{Comparison of non-impulsive forcing with the cylinder/piston mechanism}

Here it is shown that the long-duration forces discussed in $\S 2$ produce a flow which is very similar to flow produced by pushing a column of fluid through an orifice. Didden (1979) found that for short times, the trajectory of the leading vortex ring follows $x_{c} \sim t^{3 / 2}$ (up to a stroke length $L / D=0.6$ ), and this result has been confirmed in previous computations, which attempted to model the roll-up of the vortex sheet 


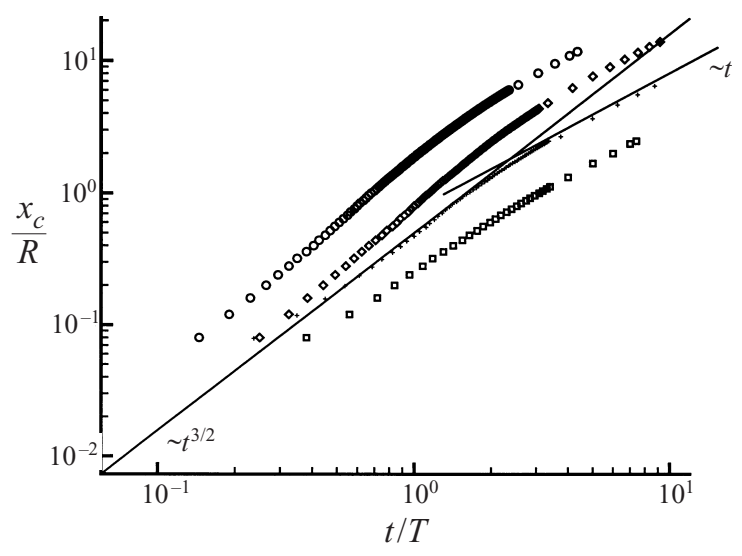

FIGURE 1. Distance of the vortex centre from the forcing location for different duration of forces. Case 1, $T C / R^{2}=1.58(\square)$; Case 2, $T C / R^{2}=4.05(+)$; Case 3, $T C / R^{2}=9.11(\diamond)$; Case 4, $T C / R^{2}=25.3(\bigcirc)$. Other parameters associated with the cases are given in table 1 .

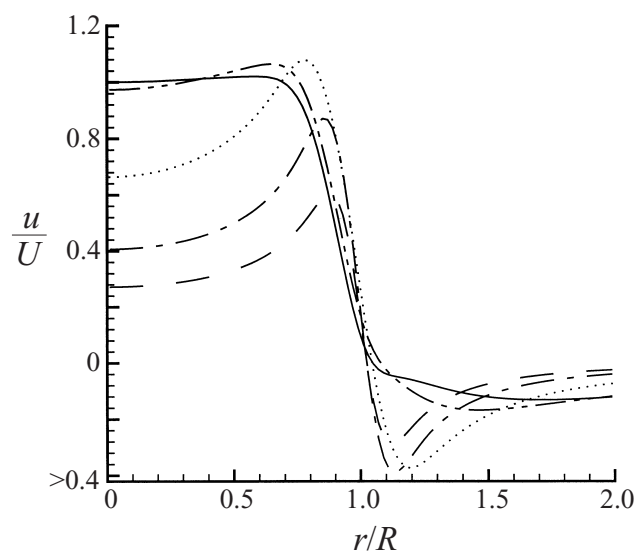

FiguRE 2. Axial velocity at the centre of the forcing region for Case 14 of table $1 . t C / R^{2}=8.86$ $(---) ; t C / R^{2}=11.4(---) ; t C / R^{2}=16.4(\cdots \cdots) ; t C / R^{2}=26.6(-\cdots-) ; t C / R^{2}=31.6$ $\left(-\right.$. The velocity is normalized with the maximum axial velocity at time $t C / R^{2}=31.6$.

explicitly (Nitsche \& Krasny 1994), and which specified an inlet velocity profile (James \& Madnia 1996). In order to demonstrate the similarity of the present generation mechanism to the cylinder/piston mechanism, we plot in figure 1 the trajectory of the leading vortex ring as the duration of the non-impulsive forcing is varied from $T C / R^{2}=1.58$ to 25.3 . For early times, the $t^{3 / 2}$ behaviour is also obtained for generation by a non-conservative force. After the forcing is turned off, the ring translates at a nearly steady velocity $x_{c} \sim t$.

The axial velocity profile at the centre of the forcing region is plotted in figure 2, at several instances during and slightly after the forcing. These plots are typical of all our runs with long-duration forces. Comparing these to similar plots from previous computations and experiments (James \& Madnia 1996; Nitsche \& Krasny 1994; Didden 1979), it is evident that the non-conservative forcing produces an axial velocity profile that has the same qualitative features as those measured at the discharge plane in the cylinder/piston mechanism. 


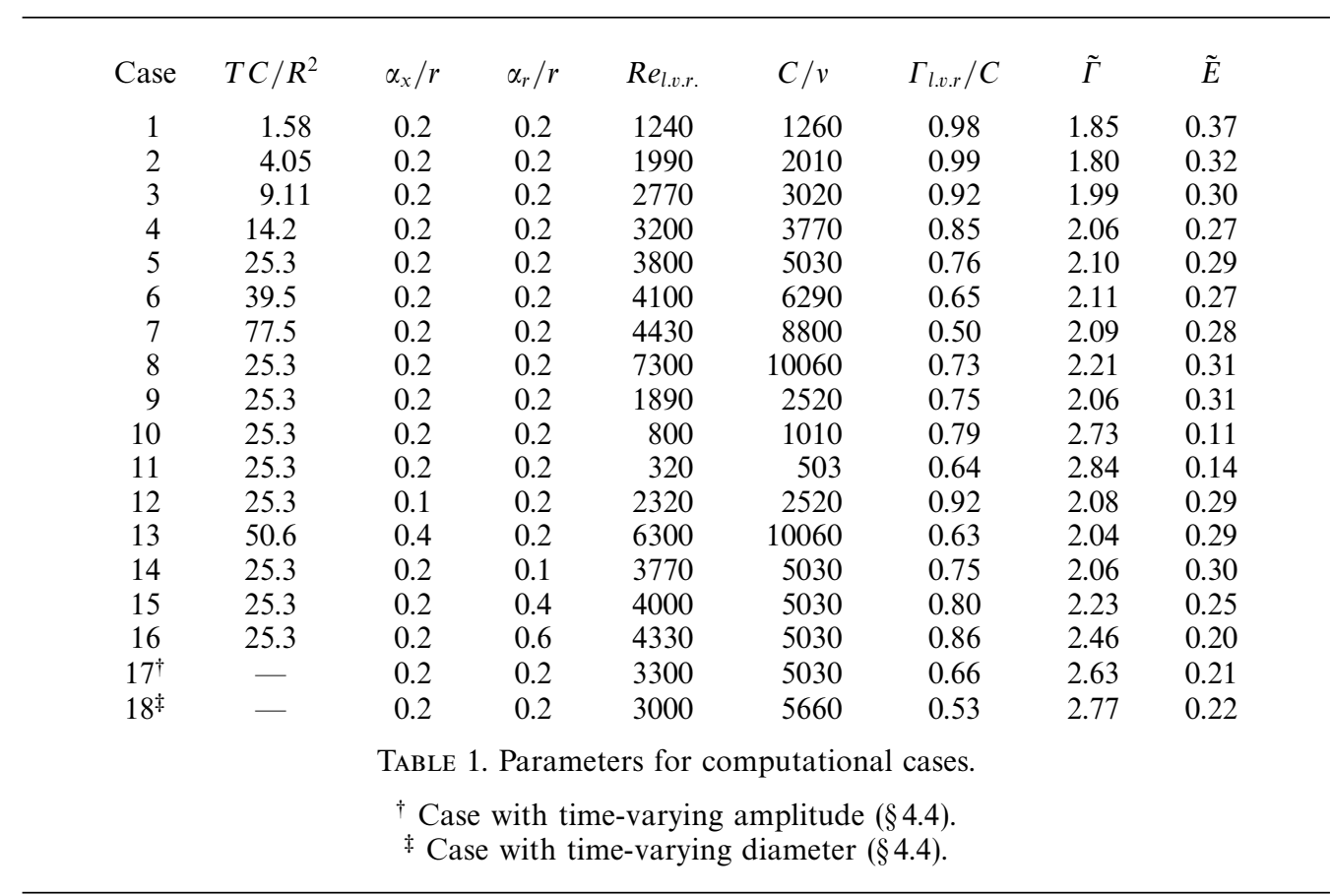

\subsection{Normalized circulation and energy of the leading vortex ring}

In this section, we vary the four parameters associated with the non-impulsive forcing and establish the conditions under which the pinch-off time and the normalized circulation energy of the leading vortex rings are independent of the values of the forcing parameters. The conditions for all the cases considered are listed in table 1 .

We begin by varying the total duration of the forcing, $T C / R^{2}$. In figure 3 , we plot the instantaneous vorticity field for three different values of the force duration at a time when the leading vortex ring has translated about 5 times the ring diameter. For $T C / R^{2}>9.11$, the leading vortex ring is pinched off from its trailing jet. In case (a), $T C / R^{2}=4.05$, all of the shear layer generated by the forcing is absorbed by the leading vortex ring and there is no visible vorticity tail behind the vortex ring. In figure $3(b)$, where $T C / R^{2}=25.3$ the leading vortex ring reaches its final configuration, while a small part of the original shear layer is left behind and not absorbed by the leading vortex. For $T C / R^{2}=77.5$ (figure $3 c$ ), there is a strong trailing jet behind the leading vortex ring. Despite the large variation in forcing times, the size of the leading vortex rings is very similar for $(b)$ and $(c)$. These figures should be compared qualitatively with figure 3 in Gharib et al. (1998), where a similar pinch-off process is observed in experiments.

In figure 4 , the total circulation and the circulation of the leading vortex ring are plotted versus time for values of $T C / R^{2}$ from 4.05 to 39.5. The normalized circulation, $\tilde{\Gamma}$, is also plotted. Several comments are in order. First, for the case with $T C / R^{2}=9.11$, the duration of the force is very close to the critical pinch-off time. For longer-duration forces, vorticity produced by the force at later times is not absorbed by the leading ring. As $T C / R^{2}$ is varied from the critical value to 77.5 , $\Gamma / C$ decreases from nearly 1 to 0.5 (table 1 ). This is a similar level of variation as found in previous computations (Rosenfeld et al. 1998). However, the normalized 

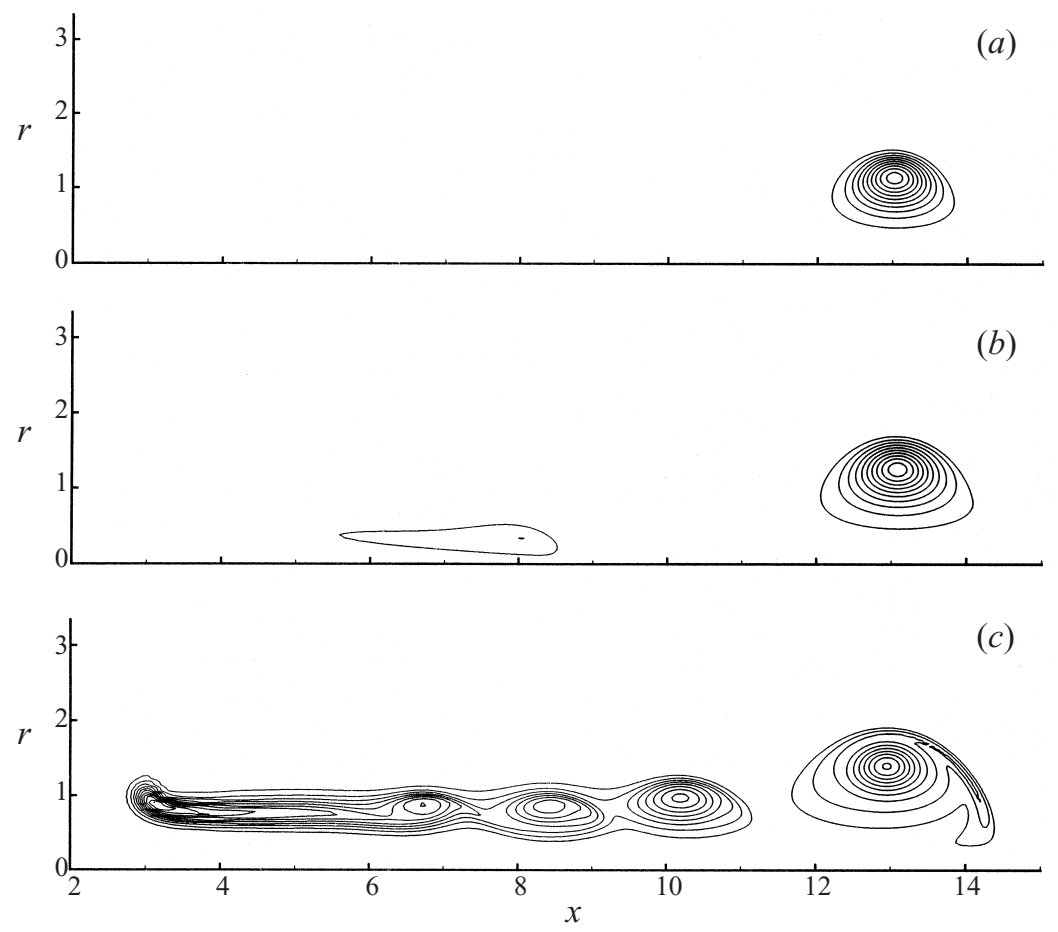

FIGURE 3. Vortex ring formation with different forcing times. (a) $T C / R^{2}=4.05$ (Case 2), contour levels $(\min =0.002, \max =0.02$, increment $=0.002) ;(b) T C / R^{2}=25.3$ (Case 5), contour levels $(\min =0.0023, \max =0.0333$, increment $=0.0031) ;(c) T C / R^{2}=77.5$ (Case 7), contour levels $(\min =0.0023, \max =0.0333$, increment $=0.0031)$.

circulation, $\tilde{\Gamma}$, is very close to 2 for these cases, with a variation of about $5 \%$, which is within the uncertainty associated with computing the integrals of the motion as discussed in $\S 3$. In the plot of normalized circulation, the line corresponds to the limiting case $T C / R^{2}=9.11$, and the constancy of the final normalized circulation for $T C / R^{2} \geqslant 9.11$ indicates that $t^{*}$ for these cases is nearly the same, with a value around 1.2. The normalized energy of the leading vortex rings is also very nearly invariant to the force duration, with values near 0.3 (table 1 ).

In a second set of computations (Cases 5, 12, and 13 of table 1), the axial extent of the forcing region, $\alpha_{x} / R$, was varied holding $\alpha_{r} / R$ constant and $T C / R^{2} \geqslant 9.11$. The parameter $\alpha_{x} / R$ has no counterpart in the cylinder/piston mechanism of vortex ring generation in the laboratory. However, for impulsive forcing, it controls the ellipticity of the leading vortex ring, which is evident from equation (2.7). In the non-impulsive case, it would appear that larger $\alpha_{x} / R$, which corresponds to smearing out the force distribution over a larger region of space, would result in a smaller percentage of the total circulation being delivered to the leading vortex ring. Indeed this was the case and for $\alpha_{x} / R=0.1,0.2$, and 0.4, we obtained circulations of $\Gamma_{l . v . r} / C=0.89,0.73$, and 0.59 , and translational velocities, $U_{t r} R / C=34,24$, and 17, respectively. Although the leading vortex ring has different translational velocity and circulation, $\tilde{\Gamma}$ and $\tilde{E}$ were again very close to 2.0 and 0.29 , respectively (see table 1 ).

Next, the radial extent of the forcing, $\alpha_{r} / R$ was varied (Cases $\left.5,14-16\right)$, holding $\alpha_{x}$ constant and $T C / R^{2} \geqslant 9.11$. This changes the thickness of the shear layer (in an analogous manner to the boundary layer thickness in the cylinder/piston mechanism). 

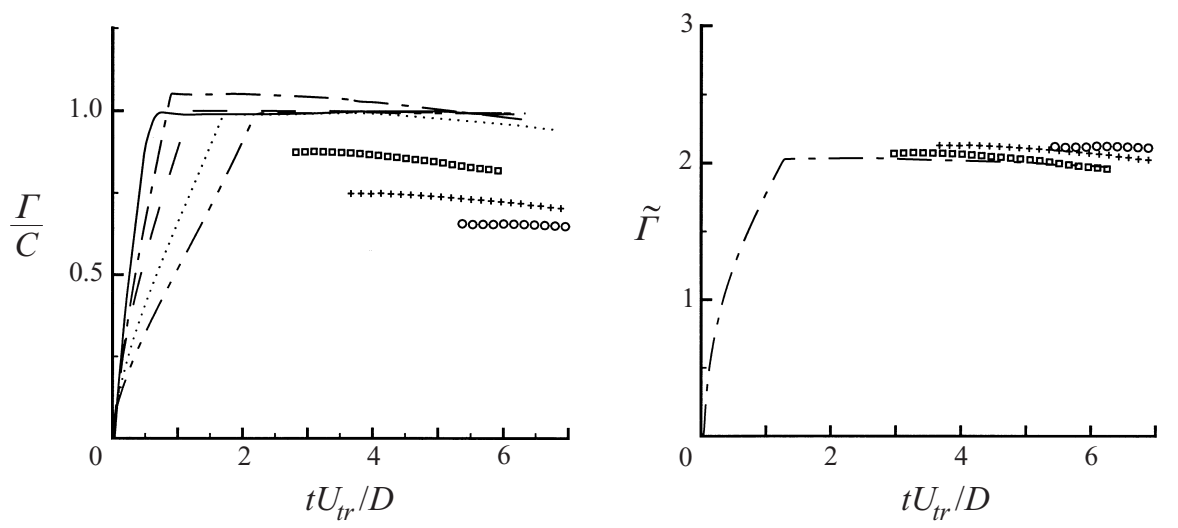

FIGURE 4. Vortex rings with different forcing duration. (a) Circulation normalized by forcing amplitude, $\Gamma / C$. (b) Normalized circulation, $\tilde{\Gamma}$. The lines correspond to total circulation, while the symbols correspond to circulation of the leading vortex ring. $T C / R^{2}=4.05$, Case $2(-)$; $T C / R^{2}=9.11$, Case $3(---) ; T C / R^{2}=14.2$, Case $4(----$ and $\square) ; T C / R^{2}=25.3$, Case 5 $(\cdots \cdots \cdot$ and +$) ; T C / R^{2}=39.5$, Case $6(-\cdots-$ and $O)$.

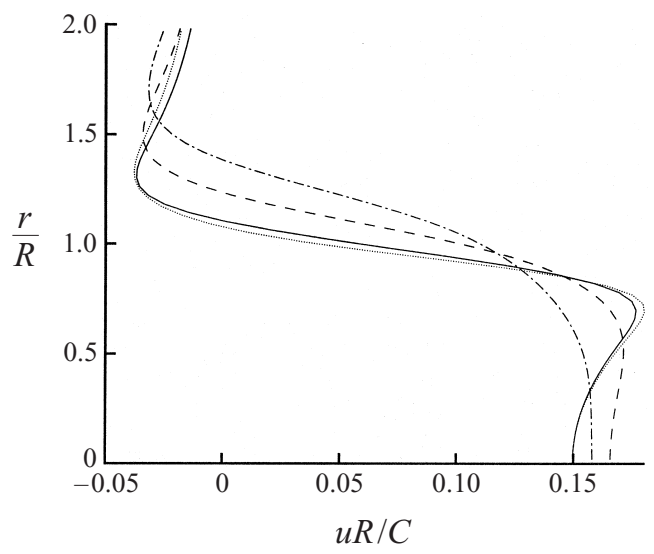

FIGURE 5 . Velocity profile at time $t U_{t r} / D=9.11$ for forces with different values of $\alpha_{r} / R . \alpha_{r} / R=0.6$, Case $16(--) ; \alpha_{r} / R=0.4$, Case $15(----) ; \alpha_{r} / R=0.2$, Case $5(-) ; \alpha_{r} / R=0.1$, Case 14 $(\cdots \cdots \cdots)$.

The axial velocity profiles at the forcing location (at a time close to pinch-off of the leading vortex ring) are presented in figure 5. For the cases where $\alpha_{r} / R$ is 0.2 or smaller, the final values of $\tilde{\Gamma}$ and $\tilde{E}$ are nearly unaffected by variations in $\alpha_{r} / R$ (see table 1). For larger $\alpha_{r} / R$ the velocity profile at the forcing location spreads in $r$. Note that at longer times, after pinch-off, these profiles become more jet-like, similar to the profile at the latest time depicted in figure 2 . These velocity profiles are similar to those computed by Rosenfeld et al. (1998), who pointed out that for constant average piston velocity the circulation generated by a parabolic velocity profile is four times larger than the circulation generated by a top hat profile, resulting in a much smaller formation number.

Finally, we varied the Reynolds number in a series of runs that produced leading vortex rings with $R e_{l . v . r .}$. from 320 to 7300 . Based on the transition map of Glezer (1988) all of these cases are laminar vortex rings. For the lowest Reynolds numbers (320 and 800) the vortex formation process was highly viscous and the leading vortex 
ring never separated from its tail at a cutoff level of $2 \%$ of the maximum vorticity. It was found that for Reynolds numbers greater than about 2000 the normalized circulation and energy of the leading vortex obtained their 'universal' values of about 2.0 and 0.3 (for sufficiently thin shear layers) indicating that the leading vortex ring reaches its final steady translation before viscosity has any significant impact.

In summary, the present results confirm the 'invariance' of the pinch-off time and normalized circulation and energy of the leading vortex ring to the details of the non-impulsive forcing, so long as the force is of sufficiently long duration, the Reynolds number is sufficiently high and the shear layers produced by the forcing are sufficiently thin. With these qualifications, the normalized circulation was around 2 , with variations of less than $10 \%$. The normalized energy was around 0.3 , with variations of less than $15 \%$. Errors associated with measuring the integrals of motion for the leading vortex ring may be a significant portion of this variation. As we have indicated, these observations are consistent with those made previously for vortex rings generated with a cylinder/piston mechanism, but we note that normalizing the circulation with the impulse and translation velocity has resulted in a much better collapse of the circulation of the leading vortex rings than has been obtained with normalization with parameters associated with the forcing (roughly $40 \%$ variation, consistent with Rosenfeld et al. 1998).

\subsection{The dynamics of the pinch-off process}

The leading vortex ring is the final outcome of the instability and roll-up of the cylindrical shear layers generated by the forcing. In the cylinder/piston mechanism, a slug of fluid is pushed through an orifice or nozzle, which creates a thin shear layer that rolls up into a spiral structure. The resulting vortex ring is 'fed' by the shear layer at a specific rate (velocity). The rate with which the integrals of motion are 'delivered' to the vortex by these shear layers would appear to vary among different vortex generation mechanisms. In the introduction, the pinch-off process was considered as a relaxation process of the leading ring to an equilibrium state. In this section, we offer an explanation for the pinch-off process based on the transitional dynamics of the leading vortex ring and its following shear layer.

The vorticity distribution is plotted in figure 6 at three different times for a typical pinch-off case. A vortex ring with small core size is generated shortly after the onset of the forcing. As the vortex ring grows in size it translates downstream due to its induction velocity. At the earliest time 46.8, which is nearing the end of the forcing time, the velocity profile at the axial centre of the forcing region, $x=3$ in the figure, resembles the velocity profile at the exit of a nozzle. At $x=5.8$, corresponding to the maximum vorticity at time 46.8 , the axial velocity profile has two local peaks. The upper maximum corresponds to the maximum axial velocity of the leading vortex ring. The lower peak corresponds to the maximum velocity in the shear layer. Since the vortex has been enlarged significantly at this stage, it pushes the shear layer toward the axis of symmetry. This is evident from the shifting of the maximum axial velocity of the shear layer toward the axis of symmetry. Since the leading vortex ring gains its strength from the shear layer, it ceases to grow if the local velocity of the shear layer is less than the velocity of the vortex at that location. We believe that this instant characterizes the pinch-off time. At time 122.7, the leading vortex is almost separated from the tail, about to absorb the remains of the tail vorticity into its core. At a later time, 217.6, the leading vortex has apparently reached a quasi-equilibrium state (slow viscous decay). This trend is illustrated in the vorticity profiles as well. The thin shear layer rolls up into a highly peaked vorticity region that accelerates 

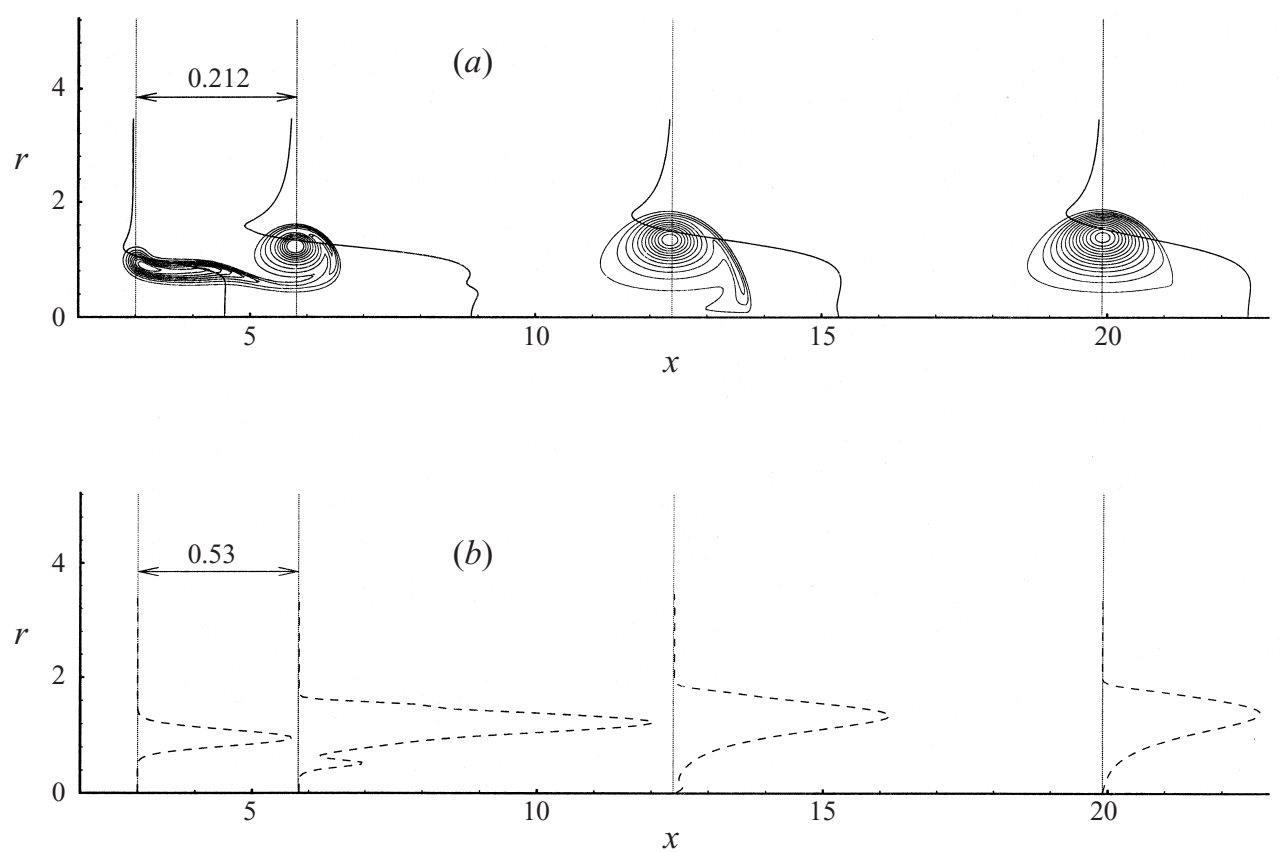

FiguRE 6. Long-duration forcing. (a) Non-dimensional vorticity contours $\left(\omega R^{2} / C\right)$ and axial velocity $(u R / C)$ profiles, and $(b)$ vorticity profiles at non-dimensional times $t C / R^{2}=46.8,122.7$, and 217.6: axial velocity $(-)$ and azimuthal vorticity $(----)$. Axial velocity and vorticity distributions at the axial centre of the forcing region at $t C / R^{2}=46.8$ is also plotted. The scales for velocity and vorticity profiles are shown.

and moves the shear layer toward the axis of symmetry. At the pinch-off time, the vorticity strength in the shear layer trailing the leading vortex tends to zero at $r=0$. From this time on, the vortex spreads more toward its final configuration where the vorticity is not highly concentrated.

This is suggestive of a dynamic criterion for the pinch-off as that time when the maximum axial velocity in the ring equals the maximum axial velocity of the trailing shear layer. Alternatively, the pinch-off time is when the shear layer is unable to enlarge the leading vortex ring, as the strength of the shear layer tends to zero at $r=0$. This suggests a means of modifying the properties of the resulting vortex ring by manipulating the characteristics of the generating shear layer. One way is to gradually move the shear layer away from the symmetry axis, so that the leading vortex ring grows away from the symmetry axis. The other is to gradually increase the velocity of the generating shear layer in order to compensate for the increasing translational velocity of the ring. These are investigated in the next section.

We note that while the dynamic approach leads to insights about the formation process, it is cumbersome for modelling the process, depending, as it does, on the nonlinear dynamics of the shear layer. The relaxation approach described in $\S 1$, validated by the apparent invariance of the normalized energy and circulation of the leading vortex ring, appears to obviate the need for such modelling, as far as the properties of the leading vortex ring are concerned.

Recently, Zhao et al. (2000) studied the interaction of the trailing jet instability (i.e. secondary roll-up of vorticity in the trailing jet) with the leading vortex ring. They concluded that this interaction accelerated the pinch-off process and was the 
cause of a $20 \%$ variation in vortex ring circulation, when non-dimensionalized with the orifice diameter and maximum piston velocity. Their implication was that this may explain the variations in circulations noted by Gharib et al. (1998). In the present computations, trailing jet instability is observed under similar circumstances (sufficiently high Reynolds number, sufficiently long forcing, and sufficiently small $\left.\alpha_{r}\right)$. However, our explanation for the variations in the vortex ring circulations is different. Our results show that by increasing the thickness of the shear layer, or by decreasing the Reynolds number, a vortex ring with a thicker core size is generated. For moderate changes in shear layer thickness and Reynolds number, however, the rate of generation of impulse and circulation was nearly constant. The thicker core size, other things being equal, results then in a slower translational velocity (this trend is also observed in the Norbury vortices (Norbury 1973)). Consequently, as discussed above, the shear layer can 'feed' more vorticity into the leading vortex ring, resulting in a higher circulation. However, as discussed in $\S 4.2$, the normalized circulation and energy of the leading vortex ring are apparently unaffected by moderate changes in Reynolds number and shear layer thickness.

\subsection{Generation of thick vortex rings}

Generation of very thick (high-circulation) vortex rings is of importance in applications, such as flow control by synthetic jets (e.g. Smith \& Glezer 1998). In the experimental set-up described by Gharib et al. (1998), and in the forcing discussed in the previous sections, it was not possible, with thin shear layers, to achieve a normalized circulation higher than about 2. In experiments, the thickness of the shear layers cannot be increased without changes to the Reynolds number or geometry of the generating device, and it is therefore desirable to seek forcing that will generate strong vortex rings, but with a small value of $\alpha_{r}$, corresponding to thin shear layers.

In $\S 4.3$ we argued that at the pinch-off moment the maximum axial velocity in the vortex ring equals the velocity of the following shear layer and that the shear layer is forced toward the axis of symmetry where it loses its strength. Therefore, one expects that if we move the shear layer away from the axis of symmetry (e.g. by increasing the orifice diameter as a function of time) or gradually increase the speed of the shear layer (e.g. by accelerating the piston velocity), it may be possible to 'feed' more circulation into the leading vortex ring. These ideas are implemented in this section.

In order to gradually increase the velocity of the shear layer, compensating for the increasing translational velocity of the leading ring, we consider a case where the amplitude of the forcing function changes linearly with time after an initial constant period. The forcing is depicted in figure 7. The normalized energy and circulation of the leading vortex ring generated by the case with variable forcing amplitude is presented in figure 8 , where it is compared with the result for a constant forcing amplitude. The normalized circulation is $2.6,25 \%$ higher than the case with fixed amplitude, and normalized energy is $0.2,40 \%$ smaller than the fixed amplitude case, which suggests that the vortex ring formed has more vorticity than the case with fixed amplitude.

Similarly one can gradually increase the radial extent of the forced region during the forcing period. In this way, at later times the shear layer will be released at greater distance from the axis of symmetry, forcing the centre of the vortex ring to move away from the symmetry axis. Consequently, while the rate of circulation generation is fixed during this process, the rate of energy and impulse delivery to the vortex ring increases. The results are presented in figure 8 where the radial extent of forcing was increased linearly for the duration of the forcing (see figure 7). Again, a very 

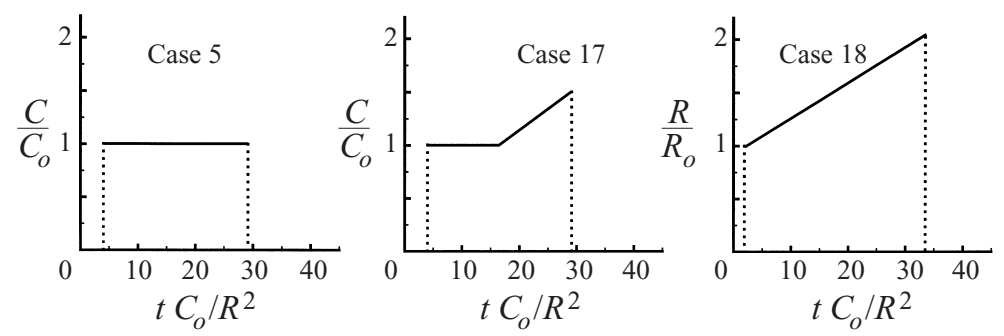

FIGURE 7. Time history of the forcing amplitude and radius for the results in figure 8.
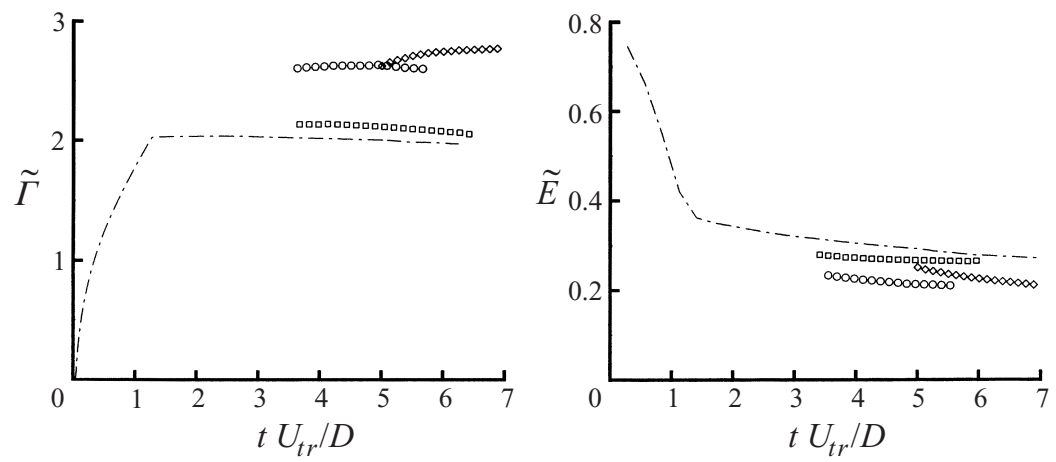

FIGURE 8. Vortex rings generated with time-varying forcing. Case $3(---)$; Case $5(\square)$; Case 17 , variable amplitude $(\bigcirc)$; Case 18 , variable radius $(\diamond)$.

strong vortex ring is produced. The values of normalized circulation and energy are comparable with similar quantities for Hill's spherical vortex.

In both numerical experiments, while no attempt was made to optimize the change in the radial extent of the forcing area or forcing amplitude with time, we were able to generate vortex rings with normalized circulation near 2.7 and normalized energy of about 0.2 , which are very close to the values of these quantities for Hill's spherical vortex, $\tilde{\Gamma}^{\text {Hill }}=2.7$ and $\tilde{E}^{\text {Hill }}=0.16$.

\subsection{Comparison with Norbury vortices}

Norbury vortices served as input to Mohseni \& Gharib's (1998) model for the formation number, as discussed in $\S 1$. Although the vorticity distribution for an experimentally or computationally generated vortex ring is quite different from the Norbury vortices, the streamlines are similar (Sullivan, Widnall \& Ezekiel 1973). In this section we compare the extent to which the normalized circulation and energy of the computed leading vortex rings are consistent with the mean core radius, as defined by Norbury as radius of a circular area that has the same circulation as a cross-section of the (non-circular) leading vortex ring.

In the computation, the area of the leading vortex ring depends on the cutoff level in defining the leading vortex ring. Changing the cutoff level from $1 \%$ to $15 \%$ of the maximum vorticity, resulted in at most $6 \%$ variation in the mean core radius. Here, all the results are presented for the cutoff level of $2 \%$. In figure 9 we compare the computationally measured values of normalized energy, circulation and mean core radius to the values for the mean core radius that would be inferred for Norbury vortices with equivalent circulation and energy. We observe that in all cases considered, the measured values are relatively close to those of a Norbury vortex, and 

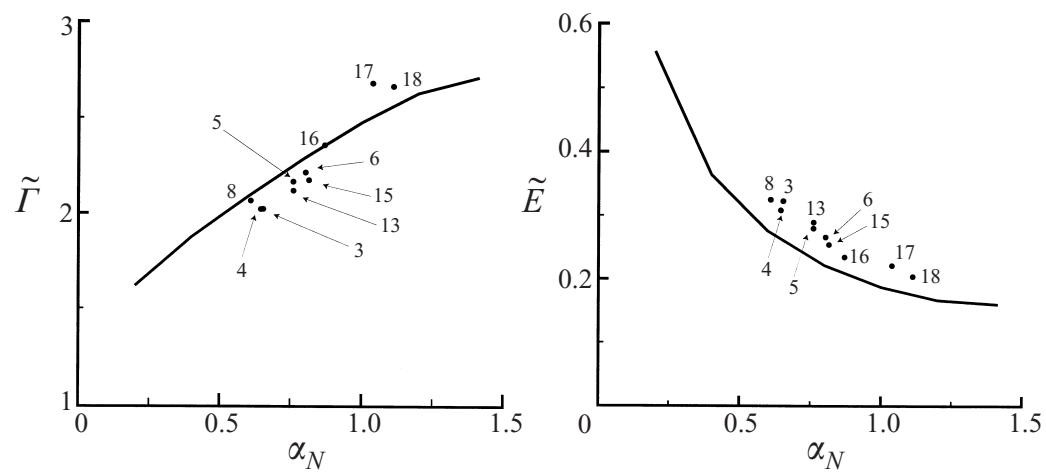

FIGURE 9. Comparison of non-dimensional circulation and energy of the computational cases with vortices of the Norbury family (-

follow the same trends with changes in $\alpha_{N}$. For cases like 15 and 16 in table 1, which have very thick shear layers that extend to regions close to the axis of symmetry, one obtains thicker rings with higher circulation and lower energy. The strongest vortex rings are generated by the mechanisms proposed in the last section. It is interesting to note, however, that even in those cases the normalized circulation and energy of the leading vortex ring follow closely the behaviour of thick vortex rings in the Norbury family.

\section{Conclusions}

Vortex ring formation by long-duration nonconservative forces was considered. An investigation of the invariance of the leading vortex ring to the forcing parameters, as observed in the experiments by Gharib et al. (1998), was carried out. The energy, circulation and impulse of the leading vortex ring were computed to determine the normalized energy and circulation as defined by Mohseni \& Gharib (1998). It was observed that if the rate of generation of the integrals of motion is constant during the forcing period, and if the shear layer is sufficiently thin (and the Reynolds number sufficiently high) the leading vortex ring pinches off with normalized energy and circulation of about 0.3 and 2.0, respectively, consistent with the theoretical predictions of Gharib et al. (1998) and Mohseni \& Gharib (1998). We show that variations in the normalized circulation are reduced to about $10 \%$, compared to variations of about $40 \%$ when the circulation is normalized by parameters related to the forcing. This latter variation is also consistent with previous computations (Rosenfeld et al. 1998) where the circulation was normalized with the average piston velocity and cylinder diameter. We note that all the results presented here were for axisymmetric, laminar flow; the extent to which turbulence alters these results remains largely unexplored.

It is suggested here that at high Reynolds number and with thin shear layers, the rate of generation of the integrals of motion needs to be modified as a function of time in order to generate stronger vortex rings with higher normalized circulation and lower normalized energy (that is, to produce thick vortex rings like Hill's spherical vortex). This statement is based on the observation that the leading vortex ring will pinch off when the generating mechanism is not capable of providing energy and circulation at a rate compatible with the normalized circulation and energy corresponding to a steadily translating vortex ring. A dynamic interpretation of the 
pinch-off process was also discussed and this motivated two ideas for modifying the properties of the leading vortex ring. In these cases we changed the radial extent of the forcing region or the amplitude of the forcing, with time (analogous to increasing the diameter of the orifice or accelerating the piston in experiments), and very thick leading vortex rings (similar to Hill's spherical vortex) were generated that could otherwise only have been created by having very thick shear layers.

In all the cases considered here, it was found that the normalized energy and circulation of the leading vortex rings were consistent with those of an equivalent vortex in the Norbury family that had the same mean core radius. It would appear that such analytical solutions provide a useful model for the energy and circulation of the leading vortex ring.

The authors would like to acknowledge many helpful discussions with Professor Mory Gharib. We are also grateful to the National Science Foundation (Grant CTS9501349) for supporting the development of the numerical simulation code used for this study.

\section{REFERENCES}

DidDEN, N. 1979 On the formation of vortex rings: Rolling-up and production of circulation. Z. Angew. Mech. Phys. 30, 101-116.

FreUND, J. 1997 Proposed inflow/outflow boundary condition for direct computation of aerodynamic sound. AIAA J. 35, 740-742.

Gharib, M., Rambod, E. \& Shariff, K. 1998 A universal time scale for vortex ring formation. J. Fluid Mech. 360, 121-140.

Glezer, A. 1988 The formation of vortex rings. Phys. Fluids 31, 3532-3542.

James, S. \& Madnia, C. 1996 Direct numerical simulation of a laminar vortex ring. Phys. Fluids 8, 2400-2414.

LELE, S. K. 1992 Compact finite difference schemes with spectral-like resolution. J. Comput. Phys. $103,16-42$.

McCormack, P. \& Crane, L. 1973 Physical Fluid Dynamics. Academic.

Miller, J., Weichman, P. \& Cross, M 1992 Statistical mechanics, Euler's equation, and Jupiter's red spot. Phys. Rev. A 45, 2328-2359.

Mohseni, K. 2000 Universality in vortex formation. PhD thesis, California Institute of Technology.

Mohseni, K. \& Colonius, T. 2000 Numerical treatment of polar coordinate singularities. J. Comput. Phys. 157, 787-795.

Mohseni, K. \& Gharib, M. 1998 A model for universal time scale of vortex ring formation. Phys. Fluids 10, 2436-2438.

Nitsche, M. \& Krasny, R. 1994 A numerical study of vortex ring formation at the edge of a circular tube. J. Fluid Mech. 276, 139-161.

Norbury, J. 1973 A family of steady vortex rings. J. Fluid Mech. 57, 417-431.

Rosenfeld, M., Rambod, E. \& GHaRib, M. 1998 Circulation and formation number of laminar vortex ring. J. Fluid Mech. 376, 297-318.

Saffman, P. 1992 Vortex Dynamics. Cambridge University Press.

Shariff, K. \& LeOnard, A. 1992 Vortex rings. Ann. Rev. Fluid Mech. 34, 235-279.

Smith, B. \& Glezer, A. 1998 The formation and evolution of synthetic jets. Phys. Fluids 10, 2281-2297.

Stanaway, S. \& Cantwell, B. 1988 A numerical study of viscous vortex rings using a spectral method. NASA TM 101041.

Sullivan, J., WidNALl, S. \& EzEKIEL, S. 1973 Study of vortex rings using a laser doppler velocimeter. AIAA J. 11, 1384-1386.

Swearingen, J., Crouch, J. \& Handler, R. 1995 Dynamics and stability of a vortex ring impacting a solid boundary. J. Fluid Mech. 297, 1-28. 
Verzicco, R., Orlandi, P., Eisenga, A., Heijst, G., van \& Carnevale, G. 1996 Dynamics of a vortex ring in a rotating fluid. J. Fluid Mech. 317, 215-239.

Weigand, A. \& Gharib, M. 1997 On the evolution of laminar vortex rings. Exps. Fluids 22, 447-457.

Zhao, W., Frankel, S. \& Mongeau, L. 2000 Effects of trailing jet instability on vortex formation. Phys. Fluids 12, 589-596. 